PSICOLOGIA Vol. XI $\mathrm{N}^{\circ} 21993$

\title{
¿POR QUE SE REFIERE A LOS NIÑOS PARA EVALUACION Y TRABAJO PSICOEDUCACIONAL? \\ Una revisión de la literatura sobre aspectos que influyen en la decisión de derivación ${ }^{i}$
}

Juana Pinzas² y Rosario Tello $^{3}$

\begin{abstract}
El artículo resume literatura norteamericana sobre factores vinculados a cuan adecuada es la derivación de casos. Se presenta tres grupos de factores: características de las profesoras, de los alumnos y de la tarea de derivación misma. Se describe cada grupo y se dan algunas recomendaciones generales con respecto a actividades previas a la decision de referir.
\end{abstract}

The article reviews North American literature on factors related to the appropriateness of referrals. It organizes them in three groups related to teacher characteristics, student characteristics and the referral task itself. Each one of them is described. General recomendations are given regarding pre-referral activities.

1 Este artículo es una revisión y adaptación de parte del informe de investigación presentado por las autoras al Summer Optimal Match Institute en la Universidad de Florida (Gainsville) sobre Diagnostics and Classroom Applications.

2 Se graduó de psicóloga en la Pontificia Universidad Católica del Perí. Obtuvo su doctorado en Psicología Educacional en Holanda. Actualmente se desempeña como Consejera-Psicóloga de Pre-escolar y Primer grado en el Colegio Roosevelt de Lima. Es investigadora en GRADE y Profesora Asociada de la Sección de Psicología de la PUCP.

3 Es Licenciada en Psicología Educacional de la Pontificia Universidad Católica del Perú. Se desempeña como Psicóloga y Profesora de alumnos con dificultades de aprendizaje en la Sección Secundaria del Colegio Roosevelt. Ha sido profesora en la Sección de Psicología de la PUCP. 

El propósito del presente artículo es examinar los factores que pueden afectar la propiedad de la solicitud de servicios especiales para ciertos alumnos por parte de las profesoras (el alumno es referido a un profesional externo para una evaluación psicoeducacional o psicológica y/o clases especiales de nivelación o remediación). Dado que una derivación apropiada es fundamental tanto para las hipótesis que guían la evaluación como para la identificación de los servicios adecuados, el tema es de relevancia. Mas aún, el tema es de prioridad si consideramos que en los colegios particulares de Lima, un número aparentemente elevado de niños es evaluado por especialistas y recibe clases adicionales después de su jornada escolar regular. Estas clases caen en una variedad de categorías: están las llamadas terapias de atención y concentración, terapias de lenguaje, terapias de psicomotricidad, terapias de entrenamiento perceptual, nivelación en el aprestamiento, repaso de matemáticas, de lenguaje, apoyo en los deberes para la casa, programas de estimulación intelectual, y una multitud de servicios mixtos no clasificables. Una característica importante de este fenómeno es que en la mayoría de los casos, los niños empiezan a recibir sus clases especiales desde muy temprano (edad pre-escolar) y, con frecuencia, continúan recibiéndolas. durante períodos prolongados.

Para lograr su objetivo, este artículo resume una revisión de la literatura norteamericana de los años 1988 a 1992 sobre factores que afectan la posibilidad de que un niño sea referido o no para evaluación y para apoyo en servicios especiales. La lectura de estas investigaciones sugiere que existen tres conjuntos de variables o factores que podríamos denominar características de la profesora, características del alumno y características de la tarea propiamente dicha. El artículo describe cada una de ellas.

\section{Los tres factores}

Cualquier referencia o derivación para evaluación o servicios especiales puede ser examinada o considerada como incluyendo tres componentes: la per- 
sona que refiere el niño, el niño que está siendo referido, y la tarea de discriminar si las necesidades del niño son del tipo que demanda evaluación especializada y servicios afines. Lloyd y Kauffman (1991) han encontrado que en los E.E.U.U. el $74 \%$ de las referencias son iniciadas por las profesoras. Sin embargo, aunque la profesora puede ser la persona que toma la decisión de referir, el niño mismo contribuye al proceso de pre-referencia pues es a sus características, conducta, aprendizaje, logros académicos, motivación, etc. a lo que la profesora está respondiendo o reaccionando. Por lo tanto, así como hay algunas características de las profesoras que las hacen más o menos proclives a referir a ciertos niños, también hay determinadas características de los niños que aumentan la posibilidad de que sean objeto de referencia. Como un tercer factor, tenemos que la dificultad de la tarea misma de discriminar la seriedad o tipo de problema escolar que exhibe un alumno y de tomar la decisión de referirlo influye sobre el proceso.

\section{Las características de las profesoras}

La investigación sugiere que las características de las profesoras pueden estar más fuertemente asociadas a la pertinencia de una referencia que las características de los colegios o de los alumnos (Meijer y Foster, 1988). Meijer y Foster identificaron la "eficacia personal" (personal self-efficacy) como una importante variable de la profesora. La eficacia personal se definía como "cuán bien los individuos creen que pueden manejar la situación o ejecutar un curso de acción (Salomón, 1983)" (en Meijer y Foster, 1988, p. 387). En los resultados de sus investigaciones los autores encontraron una asociación confiable entre el sentimiento de eficacia de la profesora y dos variables dependientes: las evaluaciones de la profesoras del grado de seriedad de un problema y la posibilidad de que el alumno sea referido. Así, las profesoras con un sentimiento de alta eficacia personal podrán sentirse más capaces de manejar los problemas escolares de un niño y se trazarán planes de acción, en muchos casos trabajando junto con los padres y adaptando sus técnicas instruccionales. Las profesoras con un sentimiento de pobre eficacia personal asumirán rápidamente que no pueden manejar los problemas del niño y requirirán la ayuda de agentes externos al aula y a la familia del niño, vale decir, de especialistas que asumirán la responsabilidad de ofrecer al niño aquella ayuda que la profesora no se siente capaz de ofrecer y/o manejar

Desde otra perspectiva, para Ruz (1990) la referencia es usualmente iniciada por la profesora en base a un juicio subjetivo. Por ello, en la fase previa a la referencia es importante el conocimiento y aceptación que la profesora tenga de la cultura del alumno. Esto es importante porque las apreciaciones que se hagan respecto a las causas del rendimiento de un alumno, tanto en el colegio como durante el proceso de evaluación, se basan en el conocimiento adquirido por el 
personal del colegio sobre la cultura de ese estudiante particular (Ruz, 1990). Esto significa que habrán algunas dificultades escolares del niño que en realidad no son tales o no son características individuales sino que más bien son en parte un reflejo o expresión de la cultura, los valores y estilo de vida del grupo familiar, social o étnico, en el que el niño ha crecido. Así, interpretar estas dificultades como expresión de un trastorno de aprendizaje puede ser un grave error. Las observaciones de Thorne (1991) cuando investigaba la lectura inicial en colegios públicos sugerían una especie de falta de disciplina que probablemente interfería con el aprendizaje y que parecía importante tratar de entender. Siguiendo el planteamiento de Ruz, es posible asociar inicialmente ese comportamiento no sólo a una dificultad del sistema educativo para estructurar a los niños y ofrecerles límites, sino también al tipo de vida que estos alumnos llevan cuando están fuera del colegio, - muchos de ellos en la calle trabajando por su cuenta (vendedores ambulantes, lavacarros, guardianes, etc.) o a cargo de responsabilidades adultas (cuidando de sus hermanos menores, cocinando y lavando para su famila, etc.). De este modo, su conducta en el centro educativo podría estar relacionada -aparte de los factores nutrición, salud y desarrollo-, ya sea a un estilo de vida sin estructuras formales o a una sobrecarga de responsabilidades domésticas que compiten con las tareas académicas y dejan una necesidad de juego y libertad.

En su estudio longitudinal de los factores que afectan el desarrollo de un ambiente de aprendizaje con restricciones mínimas (least restricted learning environment) o ambiente que limite de manera mínima las posibilidades de aprendizaje de un niño con un trastorno de aprendizaje, Rallis (1990) encontró que el entrenamiento de las profesoras y sus posibilidades de modificar la estructura de su clase de modo de poder utilizar lo que habían aprendido en su entrenamiento, eran los factores más importantes para el éxito de su enseñanza. Otros factores que afectaban el éxito del programa educativo eran: la especificidad de la profesora, su adaptabilidad, el reconocimiento de la estructura cognitiva y organizacional, y la provisión de lo necesario para un cambio real. Cuando estos factores no se daban, las posibilidades de que los alumnos fueran referidos para evaluación y servicios especiales, aumentaban. Aprendemos de esto que no es suficiente entrenar a las profesoras sino que, además, hay que apoyarlas para que puedan realmente usar lo que han aprendido, sin poner obstáculos a sus iniciativas de renovación y de cambio de estructura en su aula de clase.

Para Bauman y Frechtling (1988), la falta de entrenamiento de las profesoras con respecto a cómo manejar y trabajar con alumnos de "alto riesgo" en el aula regular, es un factor que puede aumentar el número de referencias (y la propiedad de éstas, probablemente también). Es evidente que si un alumno exhibe trastornos de aprendizaje no es suficiente referirlo a especialistas para evaluación y trabajo fuera del colegio. Si el trabajo no es en equipo, si la profesora de aula 
no introduce algunos cambios en su manera de enseñar a dicho niño y no diseña un plan de acción con metas y un sistema de seguimiento, probablemente los efectos positivos de la terapia fuera del colegio no se harán evidentes con facilidad, no se transferirán de la consulta al aula o el niño simplemente parecerá no mejorar y quedará dependiente del apoyo de la profesora particular. Para poder trabajar en equipo, la profesora de aula necesita estar bien informada y entrenada en cómo enseñar y cómo tratar en el aula regular a niños con trastornos moderados de aprendizaje. Y las profesoras o psicólogas educacionales que trabajan en forma particular necesitan asistir a reuniones en el centro educativo, responder a las demandas específicas de la profesora de algún modo y sistemáticamente ofrecerle sugerencias e informarla sobre lo que van descubriendo de la manera cómo aprende mejor el niño. Recordemos que, aunque el niño tenga una excelente profesora de educación especial fuera del colegio, él pasa todo su día escolar en manos de su profesora de aula.

Esto es exactamente lo que hemos encontrado en lo planteado por Ortiz (1992): uno de los factores o razones por las que determinados niños son referidos para servicios especiales es que las profesoras pueden carecer de estrategias de solución de problemas (problem-solving strategies) útiles para adaptar su estilo de instrucción a las características de sus estudiantes. Las profesoras, además, pueden entender la tarea de referir a un alumno para servicios especiales como una tarea individual, en lugar de usar un abordaje colaborativo con un modelo de trabajo en equipo. Cuando se usa un modelo colaborativo se da lugar a una lluvia de ideas que proporciona una variedad de alternativas para usar en el aula regular junto con la decisión de la referencia.

Finalmente, las profesoras también varían en su grado de tolerancia hacia las conductas y problemas de los alumnos (Meijer y Foster, 1988). Asi, habrá algunas profesoras que refieren a todo niño que no calza con sus expectativas de comportamiento y de aprendizaje, como habrá otras que toleran demasiadas diferencias en la conducta y en el aprendizaje, dejando pasar comportamiento y dificultades que se convierten en serios obstáculos a su enseñanza exitosa en el aula. En el primer caso, la solicitud de servicios especiales puede ser un recurso demasiado frecuente, prematuro y a veces incluso equivocado. En el segundo caso, la situación es casi opuesta, y alumnos que necesitan servicios especiales no llegan a recibirlos.

\section{Las características de los alumnos}

Ruz (1990), y Bauman y Frechtling (1988) han descrito sus hallazgos respecto a aquellas características de los alumnos que aumentan las probabili- 
dades de que sean referidos para evaluación o servicios especiales. Estos autores encontraron que el grupo étnico del alumno parecía ser una variable que afectaba las expectativas sobre su aprendizaje desde el punto de vista de la profesora y, como consecuencia, también sus posibilidades de ser referido. Bauman y Frechtling (1988) afirman que en los E.E.U.U. los negros tienen dos veces más posibilidades de ser calificados como "en riesgo" y una y media veces más posibilidades de ser considerados como con "handicaps" (limitaciones). La probabilidad de que los asiáticos sean considerados en riesgo es la mitad de la de los negros.

También es posible que la profesoras no puedan distinguir que tipo de problema exhibe el alumno (Ortiz, 1988) cuando este problema va asociado a características psicolinguísticas que ella desconoce o con las que no tiene familiaridad. Dada la dificultad para discriminar cuándo las dificultades de rendimiento académico se deben a trastornos de aprendizaje, a diferencias interindividuales en estilo de aprendizaje, y a dificultades asociadas al desarrollo psicolinguístico, las profesoras pueden evidenciar una tendencia a referir a niños de minorías linguísticas.

En el estudio llevado a cabo por Meijer y Foster (1988), los estudiantes descritos como niños que presentaban trastornos de aprendizaje, recibieron puntajes más altos tanto en grado de seriedad del problema como en posibilidad de ser referidos en comparación con los puntajes asignados a los niños con problemas de comportamiento. Así, el tipo de problema que se sospechaba tenía el niño jugaba un rol en la referencia. Es posible que en Lima, por la naturaleza del sistema educativo y por las expectativas sobre la conducta del alumno en clase, la situación sea diferente. Así, es posible que en nuestro ambiente los niños con problemas de comportamiento sean referidos más rápido y más frecuentemente que aquellos que exhiben dificultades para aprender.

Lloyd y Kauffman (1991) examinaron 382 formularios de referencia para servicios de educación especial. Estos investigadores encontraron que las razones que tenía la profesora de aula regular para referir a un alumno podrían ser categorizadas en cuatro tipos: lenguaje escrito, conducta de externalización, conducta de internalización, y problemas sensoriales.

Minner (1990) menciona en su trabajo que un aspecto relacionado a los posibilidades de ser referido para servicios especiales es la clase social del alumno. Lloyd y Kauffman (1991) señalan que un sesgo en la profesora en relación al género del alumno podría muy bien darse. Esto significaría, por ejemplo, que los niños tendrían más posibilidades de ser referidos que las niñas. De hecho, se sabe que los hombres son referidos con mas frecuencia (de 5 alumnos referidos, 4 son niños) cuando el criterio que se usa se relaciona con 
aspectos madurativos, en particular en el período de la pre-escolaridad y en los primeros grados de la educación primaria. Quizás, a la base de ello se encuentra la concepción de que las mujeres maduran más rápido lo que haría que las niñas no tengan las mismas probabilidades de ser referidas. Es interesante anotar, además, que si una creencia compartida es que los niños suelen ser mucho más activos que las mujeres, es posible que la profesora sea más tolerante con los problemas de comportamiento de un alumno que con los de una alumna.

\section{Las características de la tarea}

La tarea de la profesora antes de referir a un alumno es bastante compleja. Ella necesita asegurarse que la dificultad observada es consistente en el tiempo, necesita tratar de entender la cultura familiar y social del niño para ver si existen factores psico-sociales que interfieren, requiere tratar varias intervenciones ella misma, entre otras cosas, antes de referir a un niño. Incluso cuando estos pasos se siguen, su tarea de referir a un niño por ciertas características específicas sigue siendo muy difícil. Una confirmación de ello, puede ser encontrada en la explicación que da Ortiz (1988) en base a la dificultad inherente a la tarea de diferenciar entre dificultades de aprendizaje aparentes asociadas a estilos de aprendizaje (visual vs. auditivo, impulsivo vs. reflexivo, convergente vs. divergente, simultáneo vs. secuencial, global vs. analítico, etc.) y verdaderas dificultades de aprendizaje originadas en un trastorno.

Oakland, Shermis y Coleman (1990) desarrollaron una escala para ayudar a las profesoras en el proceso de identificar características salientes que pudieran diferenciar empíricamente a alumnos que exhiben un trastorno de aprendizaje de alumnos que no lo manifiestan. Encontraron que relativamente pocos de los ítemes de la escala tenían poder discriminatorio. Para los autores el problema proviene de muchos factores, entre ellos por ejemplo, que existen varias conductas que podrían ser típicas de diferentes condiciones o trastornos.

Cuando el alumno exhibe dificultades relacionadas a condiciones co-existentes ello puede afectar el proceso de referencia (Minner, 1990). Esto sucede, por ejemplo, con alumnos que exhiben características de trastornos de aprendizaje pero que, al mismo tiempo, dan evidencia de ser niños talentosos (gifted). Por un lado, es posible que el talento de estos niños pase desapercibido o sea obviado a causa de la etiqueta de trastornos de aprendizaje. Por otro lado, también es posible que la situación inversa se produzca y que estos niños no sean referidos a servicios para trastornos de aprendizaje, pues su talento confunde a la profesora. Es posible que ella no crea que un niño de habilidad evidentemente superior pueda tener trastornos de aprendizaje. 
Para evitar que estos conjuntos de variables tengan un efecto negativo o distorsionador sobre el proceso de referir a un niño existen algunas recomendaciones importantes respecto a la pre-referencia, es decir, respecto a qué se debe hacer antes de tomar la decisión de enviar a un alumno a evaluación psicoeducacional o a servicios especiales. Estas recomendaciones, básicamente, son: (1) revisar cuidadosamente los antecedentes del alumno; (2) llevar a cabo observaciones del alumno en el aula y en situaciones diversas; (3) entrevistarse con los padres; y (4) buscar el apoyo y sugerencias de otras profesoras del mismo nivel (equipos de trabajo). Se desprende de estas recomendaciones que existe un número de posibles intervenciones previas a referir a un alumno que son una manera de aumentar la efectividad de dicha decisión.

\section{Referencias}

Bauman, J.E. \& Frechtling, J.A. (1988). Study of the Special Education Initial Referral and Placement Process in MCps Elementary School. Eric Document $N^{o} 309619$.

Lloyd, J.W. \& Kauffman, J. (1991). Reflections on "Why Do Teachers Refer Pupils?" An Analysis of Referral Records in Exceptionality. A Research Journal, 2, 3, 157-160.

Meijer, C.J.W. \& Foster (1988). The Effect of Teacher Self-Efficacy on Referral. Journal of Special Ed., 88, 22, 3, 378-384.

Minner, S. (1990). Teacher Evaluations of Case Descriptions of LD Gifted Children. Gifted Child Quarterly, 90, 34, 1, 37-39.

Oakland, Th., Shermis, M. \& Coleman, M. (1990). Teacher Perception of Differences Among Elementary Students With and Without Learning Disabilities in Referred Samples. Journal of Learning Disabilities, 23, 8, 499-504

Ortiz, A. (1988). Preventing Inappropriate Referrals of Language Minority Students to Special Ed. Eric Document $N^{o} 309591$.

Ortiz, A. (1992). Assessing Appropriate and Inappropriate Referral System for L.E.P. Special Ed. Students. Eric Document $N^{o} 349819$.

Rallis, S.P. (1990). The Classroom Alternatives Process: School Change Policy into Practice. Eric Document $N^{o} 332277$.

Ruz, M. (1990). To Refer or Not to Refer... What Are the Questions? Evaluative Questions for Procedure Implementation of an Effective Pre-referral Phase for Culturally Diverse and Limited English Proficiency Students. Eric Document $N^{\circ} 329056$.

Thorne, C. (1991). A Study of Beginning Reading in Lima. Tesis Doctoral. Universidad de Nijmegen: Holanda. 